\title{
Reconstruction of neuronal morphologies from electron microscopy images using graph cuts
}

\author{
Huei-Fang Yang ${ }^{*}$, Yoonsuck Choe \\ From Nineteenth Annual Computational Neuroscience Meeting: CNS*2010 \\ San Antonio, TX, USA. 24-30 July 2010
}

Understanding neural connectivity and structures in the brain requires detailed 3D anatomical models, and such an understanding is essential to the study of the nervous system. However, the reconstruction of 3D geometry from a large set of dense nanoscale medical images is very challenging, because of the imperfections in staining and noise in the imaging process. In this abstract, we present an automated tracking and segmentation framework to extract $2 \mathrm{D}$ contours and to trace them through the $\mathrm{z}$ direction. The image segmentation problem is posed as Maximum A Posteriori (MAP) estimation of a Markov Random Field (MRF). The MAP-MRF formulation is equivalent to minimizing the corresponding Gibbs energy function and graph cuts [1] are used to obtain the optimal solution to the energy function. The energy function carries both the information from the current slice and from the previous slice. Furthermore, to improve segmentation accuracy, interactive segmentation editing tools that allow users to refine an incorrect segmentation are developed. Segmentation evaluation metrics using $\chi^{2}$ distance and image intensities are applied to compute confidence values of segmentations. Confidence values are helpful to point users to segmentations with low confidence values, thus reducing the time of manual inspection. Experiments were conducted on three synthetic datasets (Dataset 3 is generated by using NeuGen [3]) and on nanoscale image sequences from the Serial Block Face Scanning Electron Microscope (SBF-SEM) [2]. Table 1 shows the quantitative Dice similarity measure on the three synthetic datasets. The average DS over all experiments is 0.9113, which shows that the proposed tracking and segmentation algorithm is robust under noisy conditions. Figure 1 presents the reconstructed 3D structures of

Table 1 Quantitative Dice similarity measure of the three synthetic datasets with different levels of Gaussian noise.

\begin{tabular}{lccc}
\hline & noise $\boldsymbol{\sigma}=\mathbf{0 . 0 4 4 7}$ & noise $\boldsymbol{\sigma}=\mathbf{0 . 0 6 3 2}$ & noise $\boldsymbol{\sigma}=\mathbf{0 . 0 8 9 4}$ \\
\hline Dataset 1 & 0.9342 & 0.9315 & 0.9297 \\
Dataset 2 & 0.8948 & 0.8903 & 0.8868 \\
Dataset 3 & 0.9192 & 0.9112 & 0.9040 \\
\hline
\end{tabular}

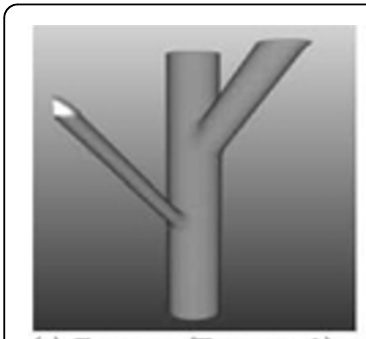

(a) Recon. (Dataset 1)

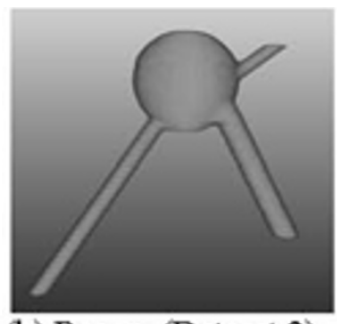

(b) Recon. (Dataset 2)

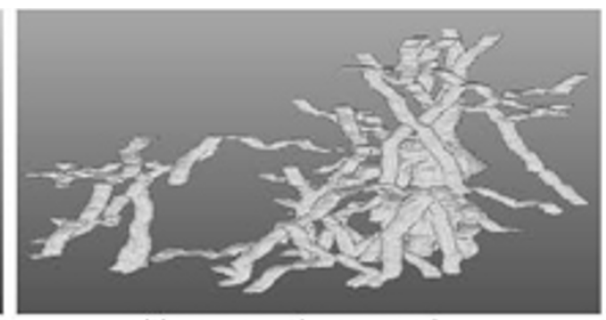

(c) Recon. (Dataset 3)

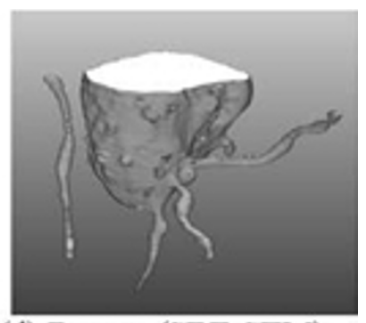

(d) Recon. (SBF-SEM)

Figure 1 Reconstruction results of synthetic datasets and of an SBF-SEM image stack.

\footnotetext{
* Correspondence: hfyang@cse.tamu.edu

Department of Computer Science and Engineering, Texas A\&M University,

College Station, TX 77843, USA
} 
tracked and segmented 2D contours, where part of a neuron can be seen in figure 1(c) and figure 1(d). The results indicate that our method can successfully track and segment densely packed cells in EM image stacks. We expect these reconstructed geometries to allow high-fidelity computational simulation of various kinds of neurons.

\section{Acknowledgements}

NIH/NINDS \#R01-NS54252 (and supplement 03S1), NSF \#0079874 (MRI), NSF \#0905041 (CRCNS), Texas ARP \#000512-0146-2001.

Published: 20 July 2010

\section{References}

1. Boykov Y, Jolly M: Interactive graph cuts for optimal boundary and region segmentation of objects in N-D images. ICCV 2001, 105-112.

2. Denk $W$, Horstmann H: Serial block-face scanning electron microscopy to reconstruct three-dimensional tissue nanostructure. PloS Biol 2004, 2:e329.

3. Eberhard JP, Wanner A, Wittum G: Neugen: A tool for the generation of realistic morphology of cortical neurons and neural networks in $3 \mathrm{~d}$. Neurocomputing 2006, 327-342.

doi:10.1186/1471-2202-11-S1-P142

Cite this article as: Yang and Choe: Reconstruction of neuronal morphologies from electron microscopy images using graph cuts. $B M C$ Neuroscience 2010 11(Suppl 1):P142.

\section{Submit your next manuscript to BioMed Central} and take full advantage of:

- Convenient online submission

- Thorough peer review

- No space constraints or color figure charges

- Immediate publication on acceptance

- Inclusion in PubMed, CAS, Scopus and Google Scholar

- Research which is freely available for redistribution

Submit your manuscript at www.biomedcentral.com/submit 